\title{
FMH-Strukturreform: Gebrauchsanweisung zu den revidierten FMH-Statuten
}

Annamaria Müller Imboden, Generalsekretärin der FMH

Hanspeter Kuhn,

Fürsprecher, stv. Generalsekretär
Korrespondenz: Rechtsdienst FMH Postfach 170 CH-3015 Bern Tel. 0313591111 Fax 0313591112 lex@fmh.ch
Die Ärztekammer hat an ihrer Sitzung vom 18./19. Mai 2006 die revidierten FMH-Statuten beraten und in der Schlussabstimmung einstimmig bei drei Enthaltungen verabschiedet [1] Sie finden die neuen Statuten auf www.fmh.ch $\rightarrow$ «über uns».

\section{Was wird wann in Kraft gesetzt?}

Der Zentralvorstand hat auf Antrag des Leitungsgremiums Strukturreform vom redaktionell bereinigten Statutentext zustimmend Kenntnis genommen [2] und beschlossen, die Statuten in zwei Etappen in Kraft zu setzen:

\section{Allgemeine Regeln und KWFB: \\ In Kraft ab 13. August 2006}

Fast alle Bestimmungen treten mit unbenütztem Ablauf der Urabstimmungsfrist (Referendumsfrist) am 13. August 2006 in Kraft [3]. So tagt die KWFB für den Rest des Jahres 2006 schon in neuer Besetzung und mit ihren neuen Kompetenzen.

\section{Die beiden Ausnahmen:}

\section{DV und GPK tagen erst im 2007}

Die neu geschaffene Delegiertenversammlung (DV) wird den Zentralvorstand in seiner Arbeit begleiten und legitimieren. Damit die DV tagen kann, müssen ihre Delegierten von den Dachverbänden nominiert und an der Ärztekammersitzung vom 14./15. Dezember 2006 bestätigt werden [4]. Dem Buchstaben nach kann die DV deshalb ihr Vorgängergremium Präsidentenkonferenz (PK) erst nachher ablösen [5]. Eine Sitzung der PK im Herbst 2006 ist aber nicht mehr vorgesehen und wurde auch von niemandem beantragt.

Die alte Finanzkommission wird abgelöst von einer Geschäftsprüfungskommission (GPK) mit erweitertem Auftrag und grösserer Besetzung. Die Ärztekammer muss auch dieses Gremium wählen [6], damit es 2007 seine Arbeit aufnehmen kann.

\section{Wer hat noch was zu tun?}

\section{Dachverbände nominieren Delegierte} für die DV

Die in der neuen DV vertretenen Organisationen - das sind: VSAO, VLSS, VEDAG, SMSR, Ordine, FMC [7], FMPP, FMCH, KHM, SFSM und MWS [8] - müssen bis zur Dezember-Ärztekammer ihre je nach festgelegter «Kuchengrösse» 1-5 Delegierten und pro Organisation einen Ersatzdelegierten nominieren, damit diese an der Ärztekammersitzung bestätigt werden und die DV ab Anfang 2007 tagen kann. Zur Erinnerung: Wählbar sind ausschliesslich Ärztinnen und Ärzte, die gleichzeitig Ärztekammerdelegierte sind.

\section{Ärztekammer revidiert die Geschäfts- ordnung}

Die FMH-Geschäftsordnung (GO) enthält die Ausführungsbestimmungen zu den FMH-Statuten. Sie wird ebenfalls von der Ärztekammer beschlossen (braucht aber keine Zweidrittelmehrheit). Leitungsgremium und ZV sehen vor, dass die Anpassungen der GO an die neuen Statuten im Herbst 2006 in Vernehmlassung gehen und an der Ärztekammersitzung im Dezember 2006 beschlossen werden können. Wir denken, dass es sich hier um eher untergeordnete Fragen handelt und keine zweite Lesung nötig sein sollte. Selbstverständlich wird die Ärztekammer darüber entscheiden.

\section{Chefärzte können den VLSS als Basis- organisation bezeichnen - sie werden angeschrieben}

Die Leitenden Ärzte und Chefärztinnen können anstelle der Kantonalen Ärztegesellschaft neu den VLSS als ihre Basisorganisation im Verhältnis zur FMH bezeichnen. Melden Sie sich bitte nicht spontan, wenn Sie sich in den VLSS als Basisorganisation umteilen lassen möchten. Sie werden angeschrieben! (Der VLSS und die Abteilung DLM im Generalsekretariat koordinieren diese Aktion in der zweiten Jahreshälfte 2006, damit alle nötigen Informationen in einem Zug zusammenkommen). 
Ein Hinweis: Auch wer den VLSS neu als seine Basisorganisation bezeichnet, muss weiterhin ebenfalls Mitglied der kantonalen Ärztegesellschaft bleiben, um auch bei der FMH Mitglied sein zu können. Das ist Teil der von der Ärztekammer nach jahrelangen Diskussionen festgelegten Kompromisslösung.

\section{Kantonale Ärztegesellschaften überprüfen bzw. revidieren Status der Chefärzte}

Die kantonalen Ärztegesellschaften sind aufgerufen, in ihren Statuten und auch bezüglich Mitgliederbeiträge die Stellung der Chefärzte zu überprüfen und gegebenenfalls anzupassen [9].

\section{Referenzen:}

1 Müller Imboden A. Protokoll der zweitägigen, ordentlichen Ärztekammersitzung. Schweiz. Ärztezeitung. 2006;87(24):1091

2 Die von der Ärztekammer am 19. Mai beschlossene Schlussabstimmungsfassung wies einige redaktionelle Inkonsistenzen auf. Wir haben diese bereinigt, damit der Statutentext möglichst klar und einfach verständlich ist. Die Textkorrekturen betrafen vor allem den VLSS und wurden in Absprache mit seiner Geschäftsleitung bereinigt. (Der erst an der Sitzung eingebrachte und schliesslich angenommene Antrag des VSAO zu Art. 8 enthielt nicht alle nötigen Folgeänderungen in anderen Artikeln zum Thema VLSS als Basisorganisation.) Wir haben den in der Ärztekammer vertretenen Organisationen zur Kenntnis eine Zusammenstellung der vorgenommenen Textbereinigungen zugestellt.
3 Das heisst: nach Ablauf der statutarischen 60 Tage nach der Publikation des Ärztekammerberichts in der Ärztezeitung; siehe Art. 24 Abs. 2 Statuten.

4 Art. 36a der revidierten Statuten.

5 Das heisst, dass die Artikel 36 bis 40 der revidierten Statuten erst ab Dezember 2006 in Kraft treten können.

6 Mit anderen Worten läuft das Mandat der im 2004 (wieder)gewählten drei FiKo-Mitglieder [siehe ÄK-Protokoll April 2004, SÄZ 23/2004; 1197] mit der Abschaffung der FiKo und der Schaffung einer GPK per Ende 2006 aus. Dies schliesst selbstverständlich nicht aus, dass bisherige FiKo-Mitglieder in die neue GPK gewählt werden.

7 FMC und SFSM gemeinsam total 5 Sitze (Aufteilung flexibel)

8 Auflistung gemäss Reihenfolge im Anhang IIb der Statuten.

9 Vgl. etwa aus dem Editorial vor der Strukturreform im Mai 2006: «Allerdings sollen die Chefärztinnen und Chefärzte weiterhin zusätzlich auch Mitglied in der kantonalen Ärztegesellschaft sein, wobei die bestgeeignete Form der kantonalen Mitgliedschaft im Kanton allenfalls neu auszuhandeln ist.» (Hanspeter Kuhn, FMH-Strukturreform vor der zweiten Lesung in der Ärztekammer, Schweiz. Ärztezeitung. 18/2006; S. 745). 